\title{
ICFA Report
}

\author{
Atsuto Suzuki ${ }^{1}$ \\ KEK, High Energy Accelerator Research Organization \\ Oho 1-1, Tsukuba, Ibaragi, 305-0801, JAPAN \\ E-mail: atsuto.suzuki@kek.jp
}

This report tells the particle physics community what International Committee for Future Accelerator (ICFA) is and does. Then, the current activity is shown with reports from ICFA Panels and discussions in ICFA Meetings.

35th International Conference of High Energy Physics (ICHEP2010)

Paris,France

July 22-28, 20

\footnotetext{
${ }^{1}$ Speaker
} 


\section{Introduction: History}

The origins of ICFA, the International Committee for Future Accelerators, go back to the late 1960s; a series of East-West meetings was held during 1967-1976 to review future perspectives in particle physics. By the end of that period, there was a belief that the next large accelerator, after the Fermilab and CERN $400 \mathrm{GeV}$ synchrotrons, would of necessity, because of its complexity and cost, be an international machine. A key meeting in ICFA's formation took place in New Orleans in 1975. Some 50 world leaders in the particle physics field passed a resolution recommending the formation of a group to study the scientific, technical and organizational problems connected with world-wide collaboration in the construction of a very large accelerator. This recommendation led IUPAP's Commission 11 to create ICFA in 1976. As might be expected, the subsequent initial decision by the U.S. to build SSC as a national machine was the subject of considerable discussion in ICFA's early days.

\section{ICFA Mission and Membership}

ICFA was created to facilitate international collaboration in the construction and use of accelerators for high energy physics. Its purposes, as stated in 1985, are as follows:

- To promote international collaboration in all phases of the construction and exploitation of very high energy accelerators.

- To organize regularly world-inclusive meetings for the exchange of information on future plans for regional facilities and for the formulation of advice on joint studies and uses.

- To organize workshops for the study of problems related to super high-energy accelerator complexes and their international exploitation and to foster research and development of necessary technology.

ICFA has a connection to other international scientific bodies through the following hierarchy: ICSU (formerly International Council of Scientific Unions, now International Council for Science); IUPAP (International Union of Pure and Applied Physics); IUPAP Commission 11 (particles and fields) and ICFA. Each entity in some way reports to the preceding one. There is also some linkage between these organizations and UNESCO.

The Committee has sixteen members, selected primarily from the regions most deeply involved in high energy physics. This has been (member numbers in parenthesis): CERN member states (3), USA (3), Japan (2), Russia (2), Canada (1), China (1), Other Countries (3). Member terms are three years, and can be renewed, generally once; term starting dates vary, in order to give continuity. Members of ICFA are nominated by designated authorities in their regions, followed by confirmation by IUPAP Commission 11. The Chair of IUPAP Commission 11 is an ex-officio ICFA member. The ICFA Chair (a three-year term) is chosen by the ICFA membership. It is not coincidental that many directors of the world's large accelerator laboratories are members, or have been members, of ICFA. For example, the current membership includes the directors of CERN, Fermilab, IHEP (Beijing), KEK and SLAC, and the DESY Particle Physics Director. Most of the recommendations about future accelerator facilities will involve existing laboratories, and any additional research for which ICFA foresees a need will almost of necessity have to be carried out at a large existing laboratory. 


\section{ICFA Panel}

Several years ago, it was realized that there are accelerator and particle physics topics of a technical nature where international discussion is valuable, and where expertise beyond that of the ICFA members is needed. Because of this, ICFA panels on specific technical topics were set up; each has about 16 members, allocated by world regions similarly to ICFA membership. Panel members are appointed by the ICFA Chair following a recommendation by the Panel Chair. Panels meet as frequently as the members decide, and reports on their activities are given at ICFA meetings. They organize schools and workshops on their specific topics and often put out bulletins, newsletters and other records of their activities. The following are the current panels.

\subsection{Instrumentation, Innovation and Development}

(Chair- Ariella Cattai, CERN)

Mission: To stimulate world inclusive involvement in the innovation and development of new instrumentation for experiments at future accelerators, and to promote research on and development of instrumentation for use in future particle physics experiments which engages physicists from all parts of the world.

Panel activities include the organization of the biennial ICFA Schools presented to advanced graduate students and postdoctoral scientists, approximately half of whom come from less industrialized part of the world than Europe, North America, or Japan, and the development and maintenance of Web-based material on instrumentation available to anyone working in the field. Included are tutorials on the various techniques as well as information on the operating characteristics of the main detector components now in use.

\subsection{Beam Dynamics}

(Chair-Weiren Chou, Fermilab)

Mission: To encourage and promote international collaboration on beam dynamics studies for present and future accelerators.

This Panel produces the Beam Dynamics Newsletter, and organizes workshops on advanced beam dynamics topics. It has three working groups, on future light sources, high luminosity $\mathrm{e}^{+} \mathrm{e}^{-}$colliders, and high brightness hadron beams.

\subsection{Advanced and Novel Accelerators \\ (Chair- Mitsuru Uesaka, Tokyo)}

Mission: To extend and support the international collaboration and communication in the field of new acceleration techniques, and to promote and encourage international collaboration/workshop/ school on advanced and novel accelerators.

This panel especially emphasizes on advanced compact accelerator and their application to not only high energy physics, particle physics, nuclear physics but also medical physics, nondestructive evaluation, security and so on, in order to maintain accelerator science and technology.

\subsection{Interregional Connectivity}

(Chair- Harvey Newman, Caltech)

Mission: To monitor and review interregional connectivity, high energy physics requirements, and make recommendations for network improvements. 
This Committee's monitoring of communications performance now extends to over 100 countries. Fig. 1 shows the correlation between the world internet density and the world night illumination.
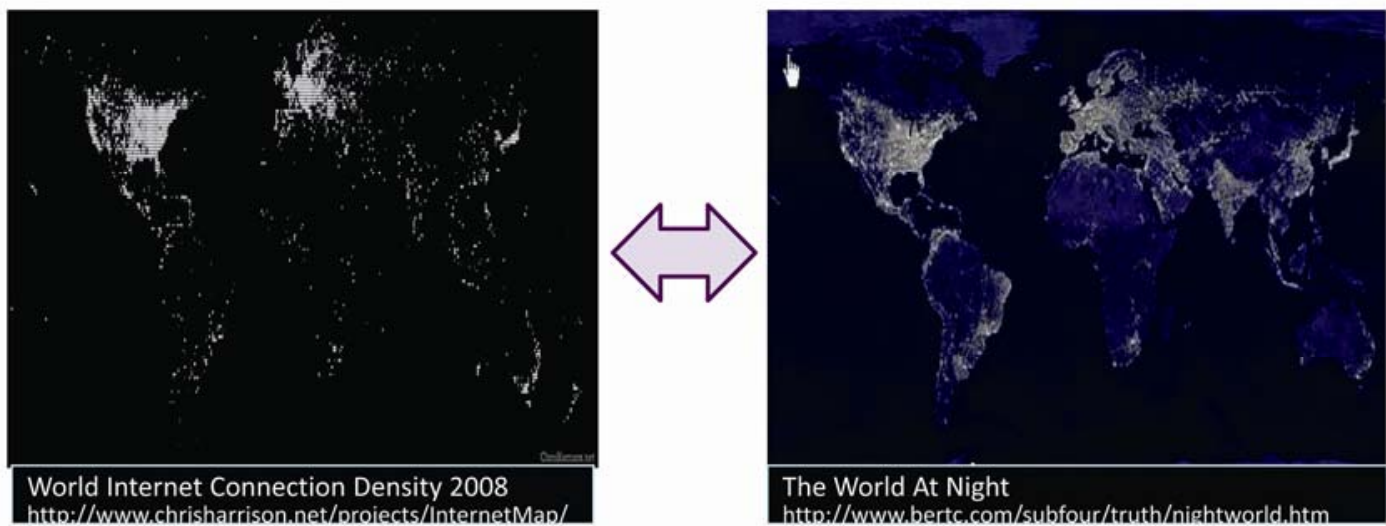

Figure 1: Comparison between the world-internet-connection-density (left) and the world-night-illumination (right).

\subsection{Particle Physics Data Preservation and Long Term Analysis in HEP (Chair- Cristinel Diaconu, DESY)}

Mission: To initiate a common reflection on data persistency and long term analysis in order to get a common vision on these issues, and to create a multi-experiment dynamics for further reference.

This panel's key-activities are to review and document the physics objectives of the data persistency in HEP, to exchange information concerning the analysis model: abstraction, software, documentation etc. and identify coherence points, to address the hardware and software persistency status, to review possible fundings programs and other related international initiatives, and to converge to a common set of specifications in a document that will constitute the basis for future collaborations.

\section{International Study Group on HEP Data Preservation}

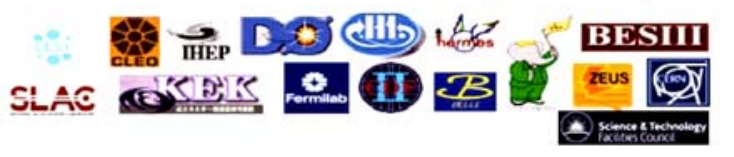

- Collider Experiments

$-\mathrm{e}^{+} \mathrm{e}^{-}, \mathrm{ep}, \mathrm{pp}^{-}$

- Computing Centers

- Some funding agencies

- About 50 contact persons
Coordination

C 1air: Cristinel Diaconu (DESY/CPPM)

Working Groups Convenors:

Phystes are François Le Diberder (SLAC)

Proservation Models David South (DESY). Homer Neal (SLAC) Technalogics: Stephen Wolbers (FNAL). Yves Kemp (DESY) Gavernances Salvatore Mele (CERN)

International Steering Committee

DESY-IT: Volker Gulzow (DESY)

HI: Cristinel Disconu (CPPM/DESY)

ZEUS: Tobias Haas (DESY)

FNALDoE: Amber Boehnlein (DoE)

FNAL-IT: Victoria White (FNAL)

D0: Dmitri Denisov (FNAL). Darien Wood (FNAL)

CDF: Jacobo Konigsberg (FNAL), Robert Roser (FNAL)

IHEP-IT: Gang Chen (IHEP)

BES III: Yifang Wang (IHEP)

KEK-IT: Takashi Sasaki (KEK)

Belle: Masanori Yamauchi (KEK), Tom Browder (Hawaii)

SLAC-IT: Richard Mount (SLAC)

BaBar: Francois Le Diberder (LAL SLAC)

CERN-IT: Frederic Hemmer (CERN)
CERN/PARSE: Salvatore Mele (CERN)

Figure 2: Data preservation activity. 


\subsection{International Linear Collider Steering Committee}

(Chair- Jonathan Bagger, Johns Hopkins)

Mission: To promote the construction of an Electron-Positron Linear Collider through world-wide collaboration. Particular attention will be given to Outreach, Science, Technology and Organization of the Linear Collider project.

The ILCSC, as a Sub-Panel of ICFA, was established in order to facilitate a global support towards the realization of the International Linear Collider (ILC) as a global collaborative effort, drawing on input from regional steering committees. The ILCSC establishes the Global Design Effort (GDE) Central Team to coordinate and direct the effort of the teams in Asia, Europe and the Americas that comprise the GDE. The ILCSC also establishes the position of Research Director, who is responsible for the development of the experimental program of the ILC. The ILCSC, representing ICFA, provides oversight to the GDE and to the Research Director. The ILCSC works closely with the Funding Agencies for Large Colliders (FALC) and/or other national or international agencies to facilitate the evolution of GDE to an institution under international governance aimed at the construction of the ILC and its detectors. The GDE Director and the Research Director are selected and appointed by the ILCSC with recommendations from the Regional Steering Committees. Both directly report to the ILCSC and are responsible to the ILCSC for leading their organizations to fulfil their responsibilities. The ILCSC assesses and endorses budget requests for the common operations fund that the GDE and Research Directors put forward to FALC for approval.

The ILCSC sets up a Project Advisory Committee (PAC) that reports to the Committee on ILC accelerator and detector issues. The PAC Chairperson is appointed by ILCSC. PAC members are appointed by the ILCSC for two years with advice from the GDE and Research Directors. The ILCSC monitors the progress of the GDE and Research Director activities, including through reports by the GDE and Research Directors and the assessment of technical progress through reports by the PAC Chairperson. The relation of these committees is illustrated in Fig. 3.

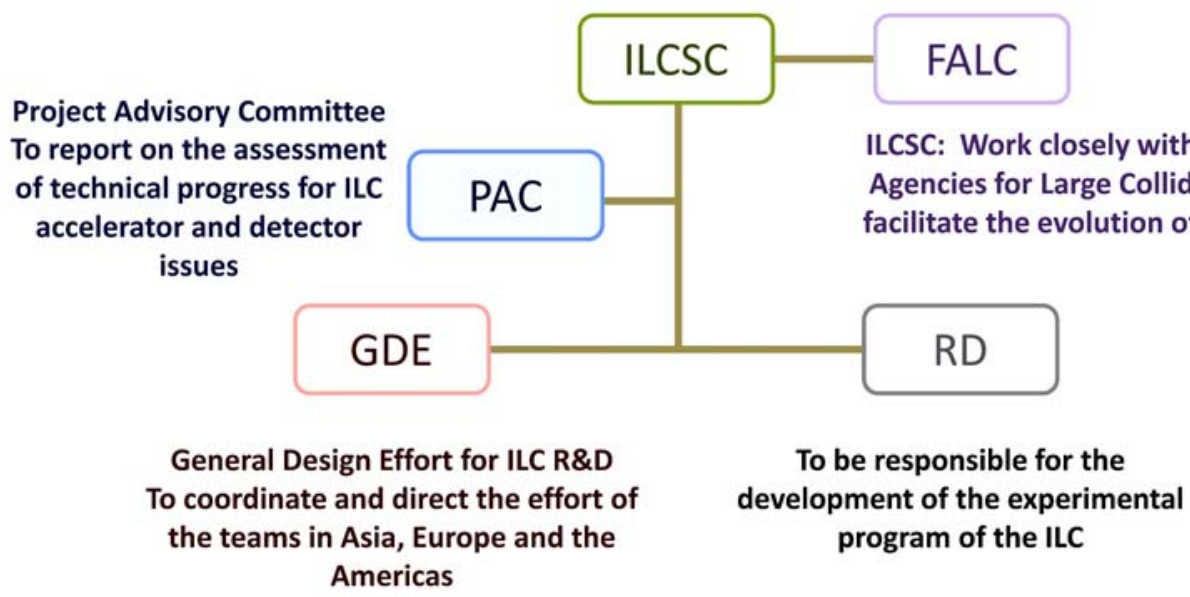

Figure 3: Oversight structure of this panel for the ILC.

The latest report from ILCSC to ICFA is itemized in the following:

- GDE keeps on track for TDR by the end of 2012, RD will produce Baseline Design for 2 detectors for TDR, it's on track, but human resource is limited. 
- Discussions occurred future governance models at the transition stage after 2012 - one viable option is a multi-national lab. collaboration (virtual lab like GDE). This becomes more real as time progresses. This governance model is general, so could cover CLIC and muon collider.

- We started a committee to write a set of ILC site requirements.

- How to evaluate successor machine to LHC needs a technical committee to evaluate readiness. ICFA organizes this evaluation.

\section{ICFA Activity}

\subsection{ICFA Meetings}

ICFA meets at a frequency (presently about twice a year) determined by the members, to consider any topic concerning future accelerators, and often related subjects such as instrumentation, accelerator technology, and also particle physics research and technology. Meetings often take place during major particle physics conferences, since many members would naturally be attending those conferences. At alternate meetings, invitations are extended to the directors of major particle physics labs who are not ICFA members; this allows for broader discussions. The Committee can make recommendations, although without any formal power to cause any resulting action. Nevertheless, the recommendations generally are at least seriously considered by laboratories and national funding agencies. Most recommendations are the result of a consensus among Committee members. A report of the most recent ICFA meeting, ICFA statements, current membership, and links to ICFA Panel pages, are on the ICFA web site. The followings are the current ICFA main topics.

\subsubsection{Particle Physics Situation --- Now and the Remainder of the Decade}

It was proposed that a vision is needed for the future of particle physics, and ICFA should produce a roadmap if necessary. Members noted the concern on how to position the field for the remainder of the decade; many things have changed in the recent past, including the LHC schedule and discussions on other projects such as neutrino facilities and B-factories. All major future facilities are counting on international collaboration, and there is a need to look at the whole picture of global projects. There are at present three existing regional roadmaps for the field, but not a global one. In the past, ICFA has generally only been involved in global, not local, projects, but since particle physics is an international endeavor, ICFA should perhaps look at the complete picture, even though it has not done in the past. ICFA is a well-suited body to produce a global roadmap, which should be physics-driven and based on the existing regional ones. It was agreed that ICFA will produce such a document.

\subsubsection{Revising the ICFA Guideline}

Since the ICFA Guidelines were written in 1980 and reaffirmed in 1993, the size, cost, and time scale of forefront particle physics projects have all increased, leading to the globalization of forefront projects. The question was raised at the previous ICFA meeting as to whether Guideline \#5 should now be revised because of these developments. The current Guideline \#5 reads:

"Operating laboratories should not require experimental groups to contribute to the running costs of the accelerators or colliding beam machines nor to the operating costs of their associated experimental areas.”

The reason for a possible change to this Guideline is that operating costs are now very large for a global project; should users be charged for running costs at a global accelerator? However, there 
needs to be sensitivity to the ability of small countries to use the facility. It was noted that new large international project designs tend to look predominantly at construction costs, not running costs.

The consensus was to add a sentence to Guideline \#5 that allocation of operating costs should be part of the agreement for building the project, and should be agreed by the parties before project approval. A proposed revision of Guideline \#5 will be presented to ICFA before the next meeting.

\subsection{ICFA Seminar}

Every three years, ICFA organizes a Seminar on Future Perspectives in High-Energy Physics, generally at one of the major laboratories; review talks are given on the state of accelerators and particle physics around the world. The Seminars typically run for several days, and have an invited attendance of $\sim 150-200$, chosen from the regions of the world using a similar formula to that used for ICFA membership; science officials from governments are also invited. The ninth ICFA Seminar was held at SLAC, on 28-31 October 2008. The next ICFA seminar will be held at CERN in October 2011.

\subsection{Joint Task Force of ICUIL and ICFA Panels}

ICFA endorsed the initiation of Joint "Task Force" between ICFA Panel on Beam Dynamics, ICFA Panel on Advanced and Novel Accelerators and ICUIL (International Committee on Ultra-High Intensity Lasers) Sub-Group on Laser Acceleration. The missions is to promote and encourage international collaboration between accelerator and laser communities for future applications of particle acceleration.

Potential next steps are to :

- Survey of the requirements for laser based light and particle sources (e.g. TeV class collider),

- Identify bottlenecks and solutions in laser acceleration physics,

- Identify bottlenecks and solutions in laser technology,

- Draft visions and roadmap, and

- Provide a global platform for intercommunity activities and communications.

\section{Conclusions}

Now that the LHC (Large Hadron Collider) at CERN has begun its continuous operation, the scientists of the HEP communities in the world are all excited by the long-awaited beam collisions. What kind of windows will open for new physics? Our expectations are mounting day by day.

\section{ICHep Congratulation on Starting the LHC Experiments PARIS/2010.}
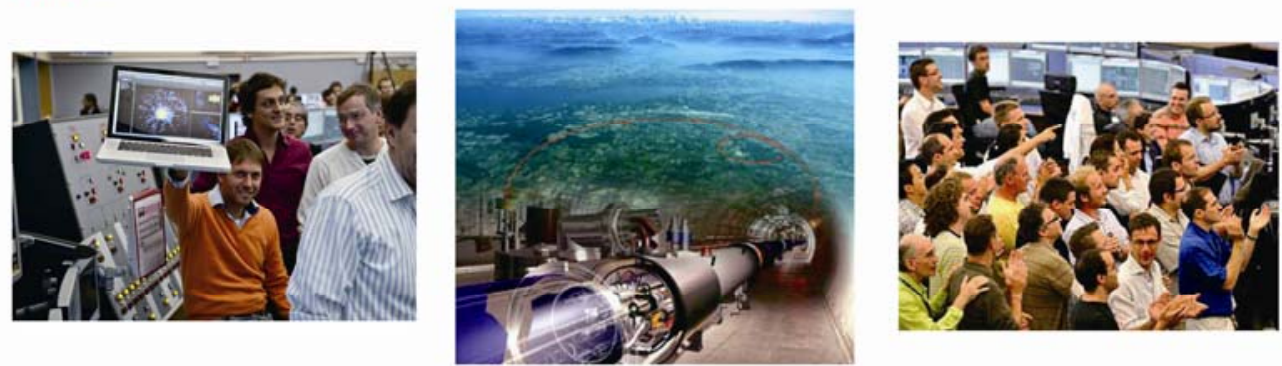

Figure 4: From the conclusion slide at ICHEP. 
ICFA plays an important role as a forum for discussions transcending national or regional boundaries on the future of high-energy accelerators and their associated particle physics, detectors and technology. It is probably true that, to paraphrase an old expression, if ICFA didn't exist, something very similar would have to be invented. Projects in our field are becoming so large and costly that no single country or group of countries can carry them out alone; more and more international discussion and cooperation is needed. ICFA will continue helping to advance the particle physics. 